\title{
NETWORK SECURITY SCIENTIFIC AND RESEARCH LABORATORY
}

\author{
Natalia Miloslavskaia, Alexander Tolstoi \\ Moscow Engineering Physics Institute (State University),milmur@mephi.edu, ait@mephi.edu
}

\begin{abstract}
Importance of Network Security Scientific and Research Laboratory in an educational process of a modern university is examined. A two-part laboratory structure and topology with respect to the initial requirements are described. Laboratory activities are discussed in detail. Designing and using of the laboratory in the Moscow Engineering Physics Institute (State University) is summarised.
\end{abstract}

Key words: security education, network security, scientific and research laboratory, distance learning

\section{INTRODUCTION}

The Information Security Faculty of the Moscow Engineering Physics Institute (State University) (MEPhI) has felt the necessity of designing new educational environment for teaching information and network technologies. A testing area for student practices today is more urgent than ever. This is not surprising as he/she has pretty high theoretical foundation and at the same time has lacked any practical training. Of course, students were taught lectures and recommended literature. Various ways of implementing the obtained knowledge in everyday practice are described. Nevertheless one has to admit that all those activities are not sufficient nowadays. When applying for a job, a person who worked with the real equipment, who designed and implemented even a small project, and more or less familiar with software in use, will undoubtedly have advantages as compared with other applicants. In fact, till now the students could gain practical experience only by themselves, mainly because it had been obtained with their own hands at the expense of other activities during their free time. 


\section{PREHISTORY}

MEPhI together with the Moscow Microsoft representatives and some Russian commercial companies (such as STC Electron-service and CROC) has already designed and implemented the Network Security Scientific and Research Laboratory. Its main goal is to implement the "education-sciencebusiness" approach in practice.

The aims of the Laboratory usage are the following:

- examination of system vulnerabilities and analysis of unauthorized access to computers and networks;

- security testing and computer-aided testing facilities;

- extending students' knowledge of security concepts and principles;

- familiarization with instruments used for ensuring system security;

- design of secure systems and subsystems;

- which, in turn, means

- a new level of scientific and research activities of the MEPhI faculty;

- an increase of efficiency of specialist training in the group of "Information security" specialties and refreshing stuff training in the field of security of information technologies, and

- adjustment of new educational technologies.

With such a Laboratory MEPhI is fully prepared to participate in the Russian federal programs on "Electronic Russia 2002-2010" and "Uniform Educational Environment". Besides it is possible not only to continue training specialists in Complex Protection of Informatization Objects, Complex Information Security of Computer-Based Systems and Computer Security, but to increase its qualitative level as well. In monthly personnel retraining courses for the Bank of Russia, Sberbank, Vnesheconombank, etc. on the basis of the faculty, results of the training with the help of expansion of practical training or carrying out extra laboratory works become more substantial.

Owing to a considerable support we are ready to put new educational technologies, for example, distance learning and progress testing and informational support of an educational process into operation.

There is an evident increase of efficiency of specialist training and progress in adjustment of new educational technologies. Students and even instructors get assistance in improving their theoretical and practical professional skills. 


\section{LABORATORY DESIGN}

Every project is fulfilled in definite stages. With limited financial resources thorough planning and design are a must for the most effective utilization of the resources and high-quality implementation of the project. All the stages were developed and realized by the students.

At the preproject stage the Laboratory design premises were explored and its necessity was motivated. The project stage followed the first one, when goals \& tasks of laboratory, objects \& methods of studies, educational courses to use it, preliminaries $\&$ knowledge and skills, configurations of the workplaces of the administrator, instructor and a typical intruder, the laboratory structure, hardware \& software, firmware requirements and specifications, educational, methodical \& technical maintenance and topics for scientific and research work were defined.

The project stage related to compiling the logical structure of the Laboratory and firmware requirements. At the same time the following main tasks of the Laboratory were worked out:

- research of the hardware, operating systems, data warehouses, software and firmware and technical means of network protection;

- design of operational models of protected networks on the basis of new information and network technologies on different platforms;

- adjustment of main methods and scenarios of distance learning and progress testing;

- information database of security technologies;

- education of users and students;

- detection of local and remote network attacks;

- analysis of mechanisms and means of attacks;

- discovery of channels of unauthorized information leaks from the system;

- definition of security policies and measures;

- elimination of consequences of unauthorized intrusion into computer systems;

- evaluation of the system protectability;

- installation, configuration and administration of security equipment;

- development of new methods and systems for information protection;

- "sandboxes" for temporary software and new technologies testing.

To carry out all those tasks successfully the Laboratory should meet some requirements. For example, when modeling secure networks it is essential to have sufficient flexibility of configuration and scalability, whereas when evaluating the system protectability and designing new methods of information protection demands a capability to react to new operational environment. The complete list of project requirements should 
include maximum flexibility, simulation of various attacks, heterogeneity, low cost and availability.

The resulting logical structure of the Laboratory satisfying all the given requirements and able to carry all the tasks is depicted in figure 1.

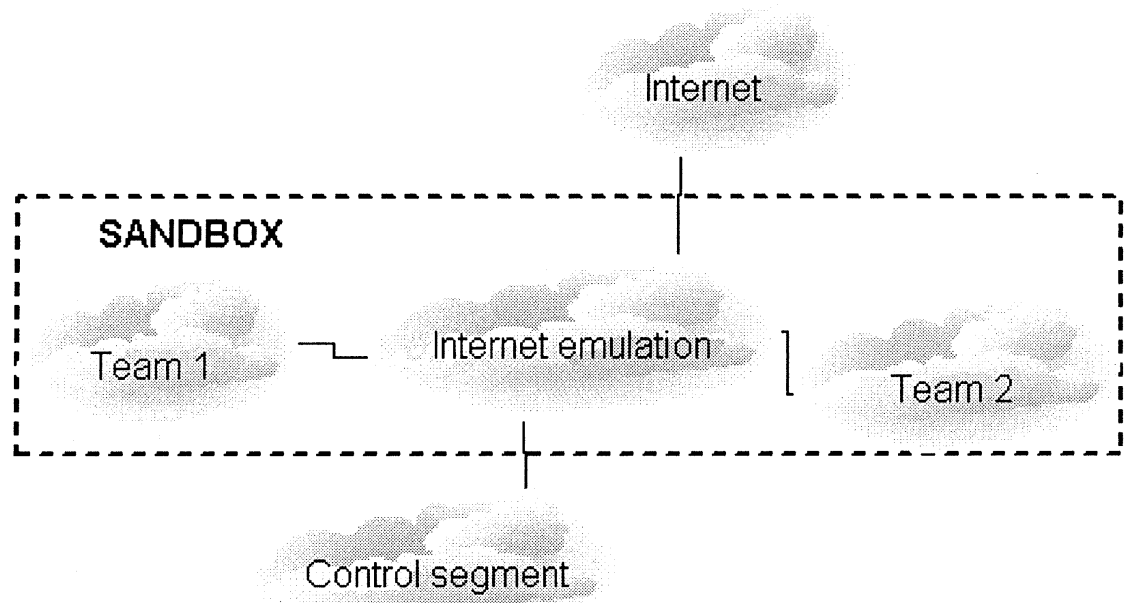

Figure 1. Logical structure of the Laboratory.

The Laboratory consists of several logical segments performing different functions which increase flexibility of the Laboratory as a whole and allows to modify it easily and/or expand it to fit new needs:

1. The Internet emulation segment, a model of public data network.

2. "Team 1" and "Team 2" segments for mutual attacks and defense.

3. Control segment, a workplace of the administrator/instructor and entrance to the Internet.

4. Transport medium, connecting all segments.

All the segments include security equipment, the specific setup being defined by the problems being solved.

The Internet emulation segment plays the role of public data network and is a transit area, passing all the traffic of participating parties, that is why it becomes a proper location for various informational warehouses, "public" servers (DNS, proxy, Web, etc.) and a management system. The management system controls operations of the segment and executes the established security policy.

The team segments simulate different corporate subnets and play the roles of networks under attack, attacking networks etc (e.g. serve as mini- 
sandboxes for temporary software testing). Accordingly the team segments should contain such firmware as:

- workstation software (OS on popular and usable platforms -Microsoft, Unix, Novell; as well as Web-browsers and other software necessary the problem solutions);

- communication facilities (they may be absent if the segment is used as an "isolated" area);

- databases (Oracle, Informix, MS SQL, MySQL, etc., the ultimate choice is defined by the problem being solved);

- e-mail facilities (servers and client software);

- different servers (application, Web-, file- and others, not yet defined);

- security subsystems and firmware security facilities;

- programming tools (for analysis of the available and design of security facilities of their own, for analysis of vulnerabilities and various technologies);

- adaptive network security and management tools, including systems for evaluation of protectability, monitoring user activity, traffic analysis and intrusion detection.

The control segment is the workplace of the administrator (an instructor participates in the laboratory works) and controls access of participants to external (relative to the Laboratory) services (for example, the Internet). This area should include adaptive network security and management tools, security subsystems and firmware security facilities, e-mail facilities and other servers.

All segments are linked into a single complex with the transport medium built on popular technologies used in networks at present. In our case the transport medium is Ethernet because it is flexible, cheap and scalable technology to satisfy nearly all the speed and quality-of-service requirements.

The team segments (and the control segment) use various software from freeware, downloaded from the Internet for analysis, to licensed operating systems and security facilities (for example, network audit tools, software firewalls, antiviral software, etc.). Besides, design and permanent update of the unified database of investigated vulnerabilities, methods of defense and used firmware, as well as maintenance of centralized support server in the control segment are of special interest.

The implementation stage follows the project stage. The faculty could not to afford a self-dependent Laboratory because of limited financial resources. That is why the next stage was the search for partners. The partners were to be interested in the Laboratory maintenance because they could shift their research and testing activities onto students and post-graduates. The partners, the Moscow Microsoft representatives, the STC Electron-Service and the 
CROC Company introduced some modifications into the initial Laboratory topology so that it would be more flexible and effective to solve various problems. The project of the Laboratory compiled by the joint efforts is depicted in figure 2.

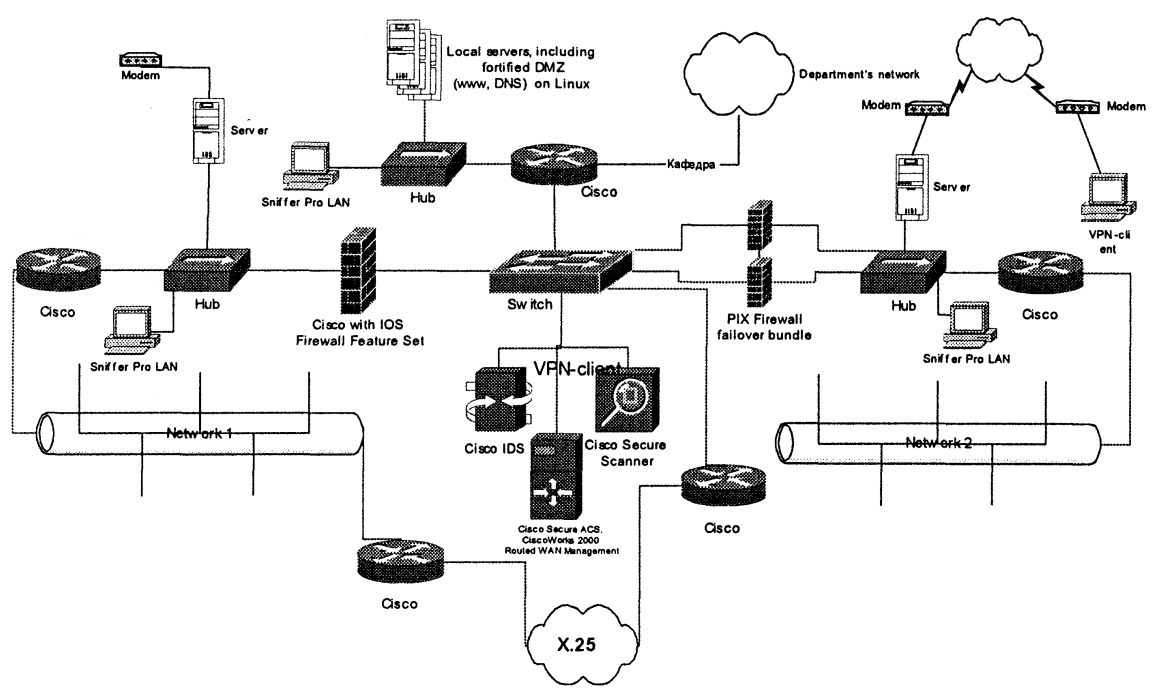

Figure 2. Laboratory topology.

After search for partners and adjustment of the project, the participants started the start-and-adjustment stage to equip the rooms, lay the communications, install and configure the equipment. Again it should be stressed that all the works were carried out only by the students.

Then the Laboratory was ready for presentation and operations test.

Figure 3 shows the Laboratory that is divided in two main parts.

The first part of the Laboratory is designed to carry out all the works listed above.

The second part is intended for improvement of the basic techniques and scripts of distance learning and progress testing. The basis of this part of the Laboratory was earlier developed at the MEPhI Distance Learning and Testing Systems (DLTS). The MEPhI DLTS is a complex of hardware, software and methodology tools for distance learning and certification based on the advanced Internet technologies and modern educational and testing techniques and specially trained personnel. Interactive DLTS Web site is constructed upon the Microsoft ASP technology. The Internet Information Server 5.0 provides the ASP support. VBScript language is used for writing ASP scripts. The DLTS information environment consists of the educational material in the HTML format and the centralized database under the 
Microsoft SQL Server 2000 control. The tools for the development of new educational course, the test tools and DLTS operation support tools are implemented as the Internet and Delphi applications. All DLTS resources must be protected and located in the Laboratory.

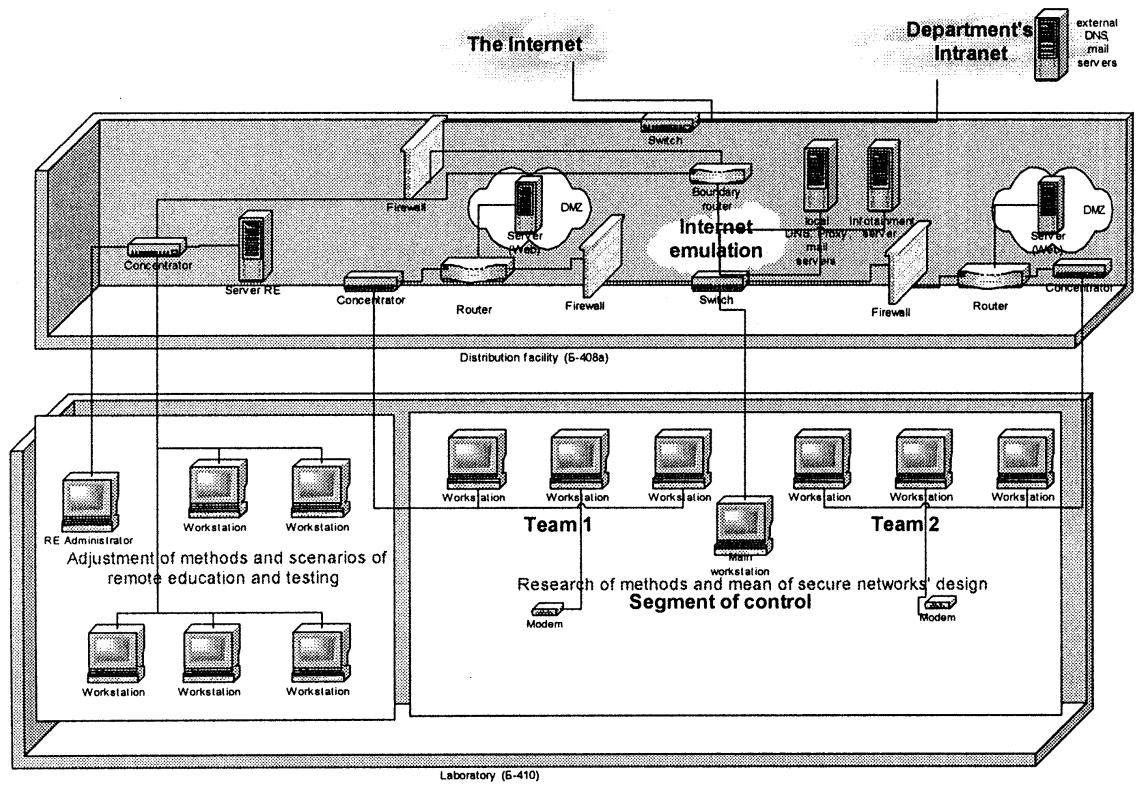

Figure 3. Equipment and configuration of the Laboratory.

\section{LABORATORY ACTIVITIES}

There is a list of requirements for trainees who work in the Laboratory: network protocols \& services, operating systems (Unix, Windows, Netware...), database management systems, computer viruses (malware), programming languages (Java, ActiveX...) and basic security principles.

The objects of the Laboratory are network hardware \& software, protocols \& services, standards, legal and norm documents, standalone computers or groups of computers in the internal and external networks with specific hardware platform and installed software - primary (for example, OS) and applied (network), with the Internet access. As for the protection hardware \& software, the students should study means of intrusion detection, security monitoring and audit, protection means (such as firewalls, encryption tools), access control implementation, security policy 
development and, of course, regulation of information security. To this end the following methods of research are used:

- emulation of intruder's activities and examination of standard attacks described in different publications;

- discovery of system vulnerabilities by scanning and probing;

- intrusion detection and elimination of consequences;

- experiments with security facilities and means of unauthorized access detection to determine the functional capabilities and to work out recommendations for installation and improvement;

- control of network information flows through traffic analysis;

- evaluation of functioning systems' protectability and recommendations for its enhancement;

- assessment of protection of computers, networks, services, protocols, hardware and software in accordance with fixed procedures and Russian standards and guidelines;

- testing system security policies and new procedures of protection to determine their comprehensiveness and validity;

- analysis of documents regulating information security;

- design, installation, configuration and administration of security facilities and patches for software and hardware.

The Laboratory participates in students training in the specialized educational courses on Information security basics, Theoretical foundations of information security, Operating system security, Network security, Database security, Complex information security of computer-based systems, Cryptographic tools of information protection, Technical methods and tools of information security, Firmware methods and tools of information security, Legal aspects of information security, Organization of information security and Building secure computer-based systems.

The subjects of the laboratory works are different. For example, the work on buffer overflow attacks is designed for the Programming technologies course, functioning of packet filters, channel encryption devices and other hardware for the Computer hardware course. Spotting leakage paths is a good illustration for the Communication networks and systems course. Analysis of OS's protectability and setup of configuration files corresponds to the Operating systems. Specific DBMS threats and built-in protection capabilities are the main topics for Database management systems. Network attacks and methods of their detection best fit Computational networks. The Management basics imply designing security policies and study of the main administrator's responsibilities, etc.

On the basis of the Laboratory there are plans to introduce the new courses on Secure network technologies, Monitoring of network security, 
VPN management, Informational and mail systems, Information security administrators, Building data networks and Network management tools.

The knowledge that trainees can gain through the Laboratory is of prior importance. For example, it allows to study the areas of:

- unauthorized computer access;

- network attacks;

- procedures and means for performing attacks;

- threats for information computer systems;

- vulnerabilities and bugs in systems, services and network protocols through which the adversary's intrusion can be expected;

- channels of unauthorized information leaks from the system;

- network security monitoring;

- access isolation systems providing a controlled access to informational and network resources;

- secure informational systems;

- system's security policy;

- measures and procedures of accident prevention;

- eliminating the consequences of unauthorized intrusion into a system;

- system protectability;

- defining the purpose, basic functions and place of information security standards in the system; usage peculiarities of the specific standard;

- evaluating the functional capabilities of the existing security equipment and determine the applicability of firmware in network architectures;

- configuring the security facilities built into many systems;

- ensuring the secure operation of system applications;

- administering security equipment;

- methods of defense;

- implementing new systems and means of information protection;

- basic regulations and standards in information security;

- preparing documentation for a new security equipment for further state level certification.

This is not a comprehensive list. Every student chooses his/her own specialization and questions that he/she will thoroughly study. It is impossible to be a specialist in every possible field. Nevertheless all students can obtain a necessary minimum of knowledge in their own problems mentioned to continue independent studies and research.

On the basis of the typical basic tasks as well as our personal experience the following scientific and research topics were suggested as subjects of student's research.

\section{Design of a Network Security Scientific and Research Laboratory} (complex project): Goals, tasks, and requirements. Hardware and software. Laboratory structure. Objects and methods of research. 
2. Laboratory equipment: Marketing research. Start-and-adjustment works.

3. Laboratory maintenance: Educational and methodical maintenance. Information support, resources \& interfaces. Glossary. Intruder's models and workplace of an attacker. Workplace of network security administrator. Instructor's interface for dynamic reconfiguration of Laboratory depending on the attack under study. Instructor's interface for monitoring Laboratory operations.

4. Laboratory practice: Emulation of specific attacks. Emulation of secure network protocols. Interfaces emulating operation of security facilities. Network topology _ attacks \& Transport medium in use _ attacks dependencies. Peculiarities of telephone channel \& fiber-optic attacks. Peculiarities of attacks from the Internet. Attacks on network hardware \& software. Vulnerabilities and protection of workstations. Attacks on firewalls \& proxies. Vulnerabilities of client/server architecture. Vulnerabilities of databases and database management systems. Attacks on electronic document interchange. Vulnerabilities and protection of Web-servers and applications. Application-level attacks and protection. Vulnerabilities of network services and commands. Crypto protection. Digital signature. PKI. Firewalls. Adaptive network security. Antiviral software. Virtual private networks. Protection against an unauthorized access. Security policy development and management. File and session encryption. Network-based \& host-based intrusion detection systems. Attacks on IDS. System \& network security scanners. Security services: intrusion tests. Trusted operating systems. Design of own means and methods of defense.

\section{CONCLUSION}

Development of Network Security Scientific and Research Laboratory allows not only to improve student's training in the set of information security specialties, but it also raises educational and research activities of the faculty to a new standard which results in increased efficiency of training and retraining courses on old and new educational programs and participation in the Russian federal programs. It permits online exchanges of experience with foreign partners and to joint investigation and research (the so-called exterior projects). With a mutual agreement it is possible to participate even in the joint laboratory works when, for example, Russian, American [1], Australian [2] or Greek [3] students can compete in network protocols, technologies and network protection tools. 


\section{REFERENCES}

[1] White G.B., Sward R.E. Developing an Undergraduate Lab for Information Warfare and Computer Security. Proceeding of the IFIP TC11 WG11.8 First World Conference on Information Security Education. 17-19 June 1999, Kista, Sweden. Pp. 163-170.

[2] Armstrong C.J., Armstrong H.L. The Virtual Campus. Proceeding of the IFIP TC11 WG11.8 Second World Conference on Information Security Education. 12-14 July 2001, Perth, Australia. Pp. 161-168.

[3] Gritzalis D., Tryfonas T. Action Learning in Practice: Pilot delivery of an INFOSEC University laboratory course. Proceeding of the IFIP TC11 WG11.8 Second World Conference on Information Security Education. 12-14 July 2001, Perth, Australia. Pp. 169182. 